\title{
Proposal Submission System - A Content Management System Approach for Proposal Submission
}

\author{
Saeed Shadlou, Chai Kinn Pong, Sanath Sukumaran \\ Taylor's University
}

\begin{abstract}
Submissions of proposals are very often a tedious affair. Proposals which are submitted goes through not only a complex and tedious process but often the scrutiny of many individuals of different roles. Hence, it is important that any automation envisaged goes through a meticulous exercise to achieve the same. Proposal Submission System aims to provide an online proposal submission mechanism, improving the traditional and physical methods of submission. Such system allows assignment submission to be more efficient and greater rigor. Online submission provides electronic copy of assignments, which has many benefits over traditional physical copy submission of assignments. With the implementation of Proposal Submission System, one can be subjected to the same rigor as traditional means without compromising on quality whilst adhering to the same process workflow.
\end{abstract}

\section{CONTENT MANAGEMENT SYSTEM}

Traditional paper based submission had introduced various problems to academic staff and students. Online submission provides flexibility for submission regardless of physical location, save up physical space by using electronic copy of submission and ease evaluation process of academic staffs. Email provides a solution towards proposal and other documents' submission online. However, organizing submissions in email system is time consuming and inefficient. A online web content management approach to handle the activities of creation, submission and approval will be more beneficial.

Proposals are widely in use under various occasions. Type of proposal includes project proposal, system proposal, research proposal, business proposals, etc. In academic field, academic proposals are the first step in producing research papers, thesis, or major projects.

A content management system is a database of information and a way to change and display that information without spending a lot of time dealing with the technical details of presentation. [3] CMS as an effective media allow users to share and manage information easily through the use of web browsers. Many had utilize the advantages of CMS in various situation. Organizations use CMS to store, display, and organize information. Other forms of CMS include personal blogs, library portals, development wiki and etc. 
International Journal of Web \& Semantic Technology (IJWesT) Vol.2, No.2, April 2011

A CMS approach for the system allows creation, editing, submission, and approval of proposal on a website without any need of third party software. The CMS will also able to track any updates and status of each proposal, allow notification to be send to related parties.

With the use of CMS, academic staffs will be able to manage the flow of proposal submission and approval activities in a more organized manner. Student will go through a proper procedure to create a proposal and this ensure the proposal consist of all required details before submission for approval. This provide a consistent format or layout for proposal submission, ease the evaluation process of academic staff on approval of proposals.

\section{THE PROPOSAL SUBMISSION SYSTEM}

The objective of Proposal Submission System is to provide an online web based solution for academic use. The system covers complete process of proposal submission and approval, from creation and submission by participants, to comment and approval by coordinator. Functionality regarding user profile, help and frequently asked questions, contacts and feedback are among the functionality of the system.

The users of the Proposal Submission System are group into 2 party, the participant and coordinator. Participants are personal which create and submit a proposal for approval, mainly current student of the university. Coordinators are academic staffs, which will receive, evaluate, comment, approve or reject proposal submitted to them. Both party require registration to the system, providing respective ID to verification as valid user of the system. As the evaluators of proposals, coordinator plays a more important role in the system, the registration of coordinator require approval from system administrator. An email notification system will be in place to complete registration of both party.

Create a proposal is the first step in the process of proposal approval. Proposal Submission System plans to include a step-by-step proposal creation form, to assist participant on creating a proposal for submission. An optional field for file attachment is available, allowing user to attach relevant documents for proposal submission. Upon completion of all steps, following will be submission of proposal to selected recipient from the list of registered coordinator.

After submission of proposal, participant will be able to view the proposal in his/her user profile, with a pending status under list of proposals. Coordinator will be notify of the submission of the proposal, and coordinator's user profile will also be updated to reflect the receival of proposal. A participant will then able to view the proposal he/she submitted, download the attachment or cancel the submission if the submission still within 24 hours (or any time specified by system admin). Coordinator will be able to view the proposal as well, with additional option to comment, approve or reject the proposal. 


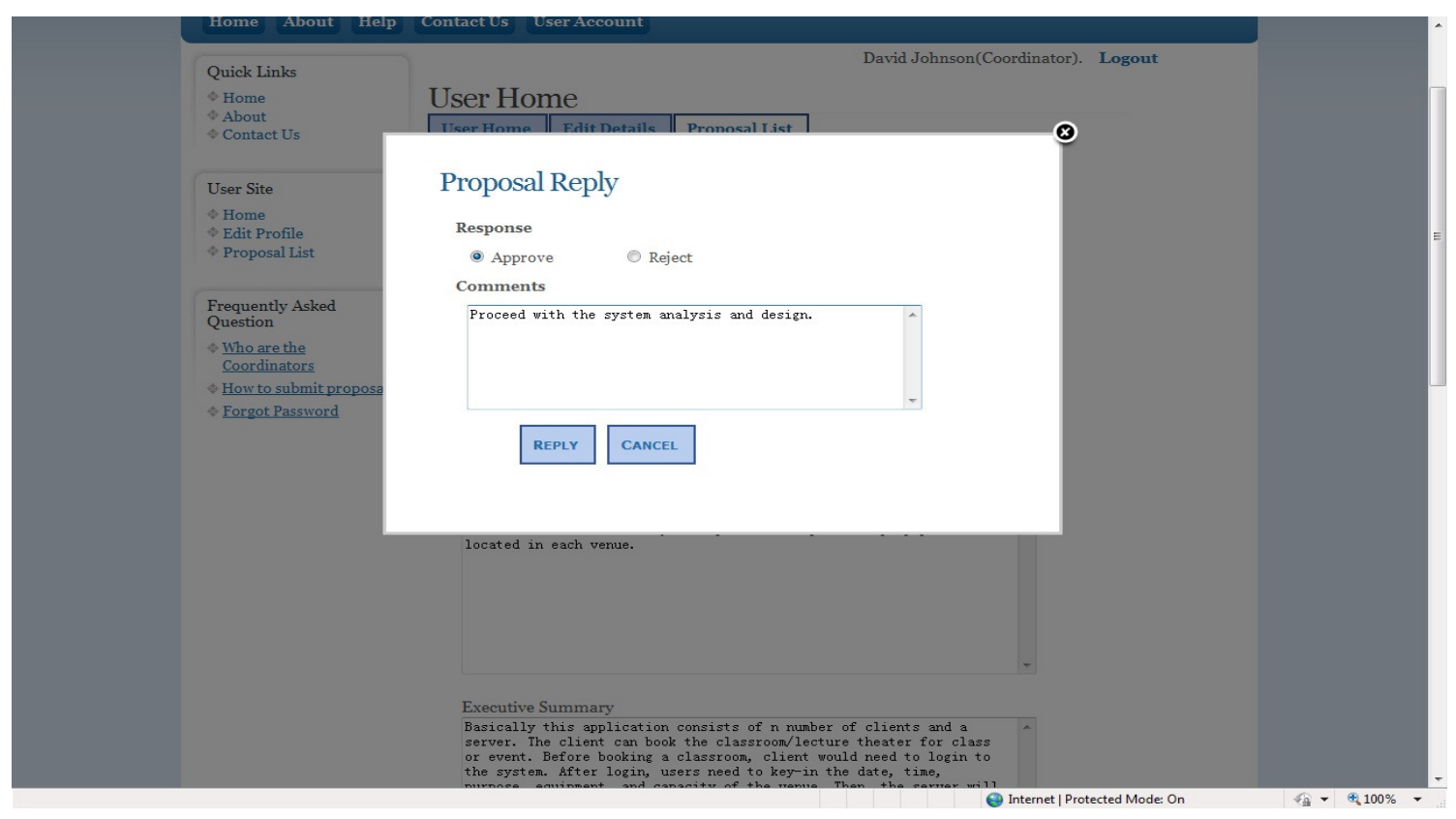

Figure 2(Proposal Reply)

Email notification system will be used to notify users on any alert raised from the system. Possible situation where email notification will be trigger including coordinator receive a new proposal, participant's proposal being accept or rejected, coordinator comment on participant's proposal. Email will also be send during registration stage, as the last step to complete registration.

\section{METHODOLOGY \& TIMELINE}

The developer had chosen to practice Hyperlink Web Development Methodology [9], and the development timeline is created base on the methodology. The timeline diagram covers analysis and design work done in the early stages, time frame of developing and testing the system, and deployment date.

This timeline diagram shown is the initial development timeline created during the earlier stage of the system design phrase. With the development timeline as the guide for completion of tasks, the system development is to be completed in time. 


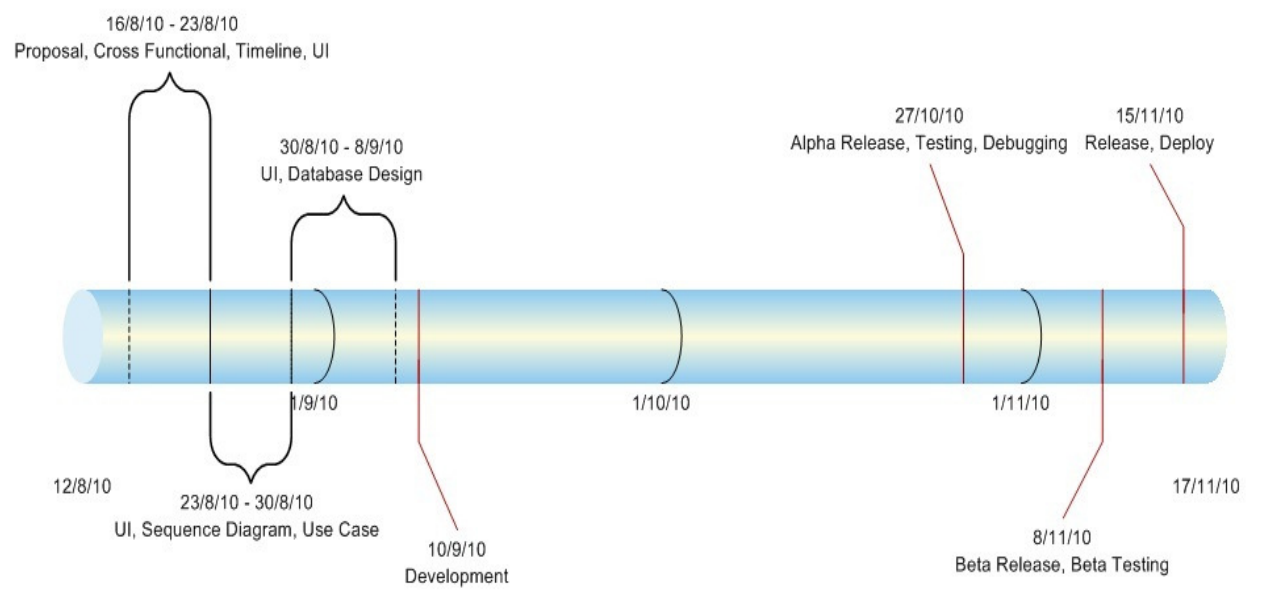

Figure 3 "Development Timeline"

\section{TOOLS AND TECHNOLOGY FOR DEVELOPMENT}

There are many options and choices in term of web development tools, platform, system and technology. Few of the major web development system available will be the AMP solution stack, Microsoft's .NET framework and Java's J2EE [4]. Among the choices, AMP package stands out as a popular option of free open-source system available for development.

AMP package consist of Apache server, MySQL database with PHP, Perl or Python as server side scripting language. Developer had chosen AMP for development of Proposal Submission System due to his experience with development with AMP package with the ease of installation with XAMPP. XAMPP is a full featured AMPP ( Apache, MySQL, PHP, Perl) package that is one of the few non-commercial AMPP middleware stacks available cross platforms. It's packages includes not only AMPP, but also database tools (phpMyAdmin, SQLite), PHP packages (PEAR) and other development tools. With its features, it is possible for XAMPP to be use for personal home page to production site for developments [5].

JavaScript mentioned to be the most popular scripting language on the internet in w3schools's JavaScript section, which works with all major browsers includes Internet Explorer, Firefox, Chrome, Opera and Safari [6]. AJAX (Asynchronous JavaScript and XML) introduced the ability to compose asynchronous background requests to the web, from the client machine. This allow applications to provide rich client-side interfaces, and allows browsers to communicate with the web without forcing page refreshes; both fundamental features of Rich Internet Application. AJAX supports thin client applications that can take full advantage of computer power of clients, reducing the server load [7].

Development of JavaScript involves usage of library, especially when comes to cross-platform and cross-browsers compatibility. JQuery is one of the high-quality, open-source JavaScript tool kit, including large number of ready-made plugins, many that provides user interface functionality [8]. 
International Journal of Web \& Semantic Technology (IJWesT) Vol.2, No.2, April 2011

\section{SYSTEM FUNCTIONALITY}

Based on requirement specification, the cross-functional flowchart is created and functions of each requirement are linked in the chart. This produced an overview of activity flow of the Proposal Submission System with relationship between different function. The cross-functional flowchart is produced and further improved.

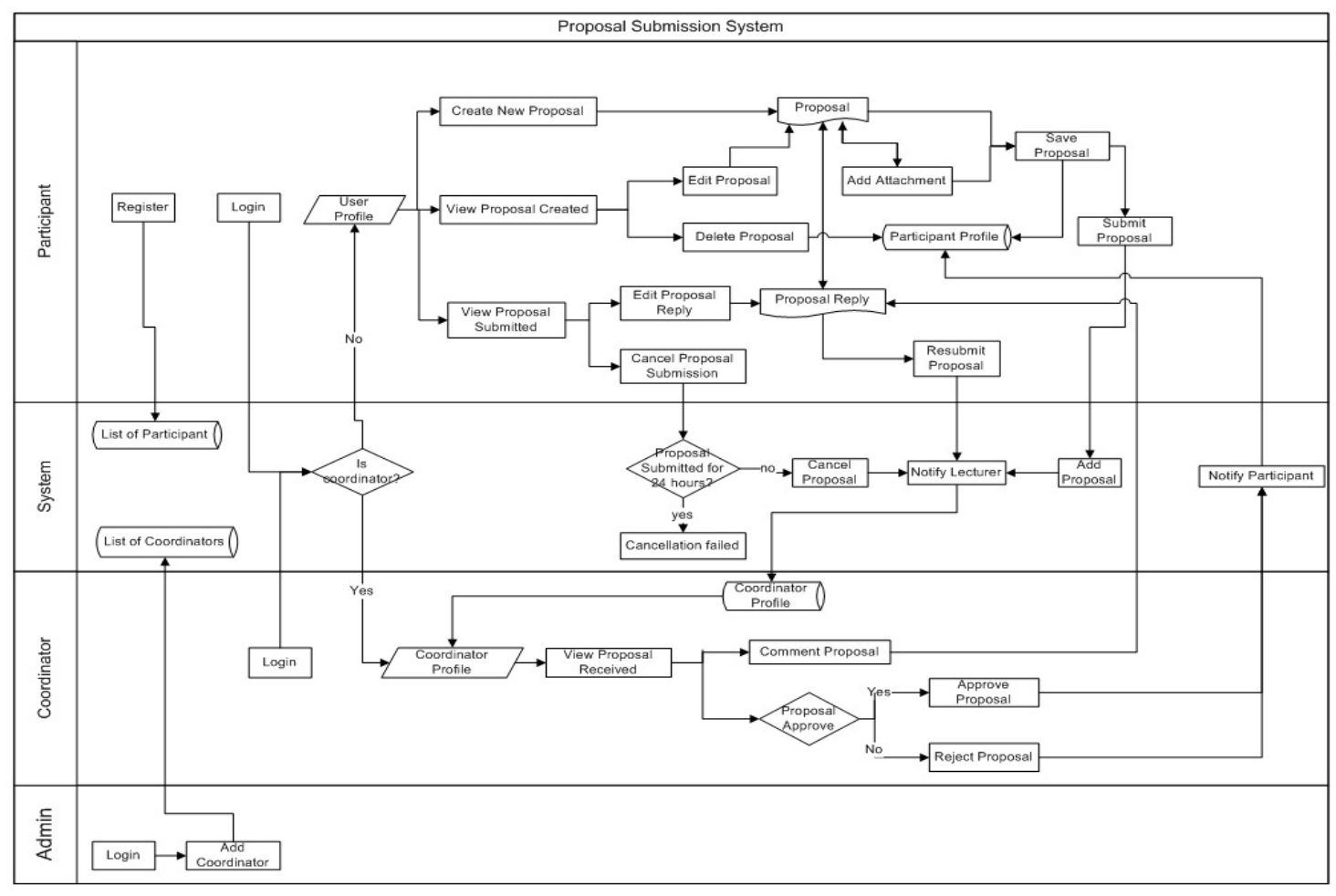

Figure 5 "Cross-Functional Flowchart"

The flowchart had specified the interaction between different users with the system, and mapped the communication between functionality of the system. The Proposal Submission System is to be implemented according to this cross-functional flowchart.

\section{SYSTEM REQUIREMENT}

Requirements of the Proposal Submission System can be segregate into categories based on 3 different types of users of the system: participant, coordinator and admin. Each of the users will interact with the system in different aspect and different level.

\section{Participant}

Participants are undergraduate students of Client University, which required to submit academic proposal to academic staff for their coursework. They are registered users of the system, able to access the following functionality: 
International Journal of Web \& Semantic Technology (IJWesT) Vol.2, No.2, April 2011

- a user home page, which consist of participant details, proposal statistics, and a few of proposal created;

- user profile page, allow participant to change and update their information, include changing email and password;

- proposal list, a list of proposal created by participant, sorted with creation date of proposal and with status of proposal shown;

- create new proposal to be submit to coordinator;

- view a proposal created by participant;

- receive notification on proposal updates from coordinator, such as approval, reject or comment;

- Cancel a proposal submission, if submission time within past 24 hours (or duration specified).

Participant provided with functionality of profile management and proposal submission. This is due to the involvement of participant in the system are proposal submission and awaits coordinator response regarding proposal.

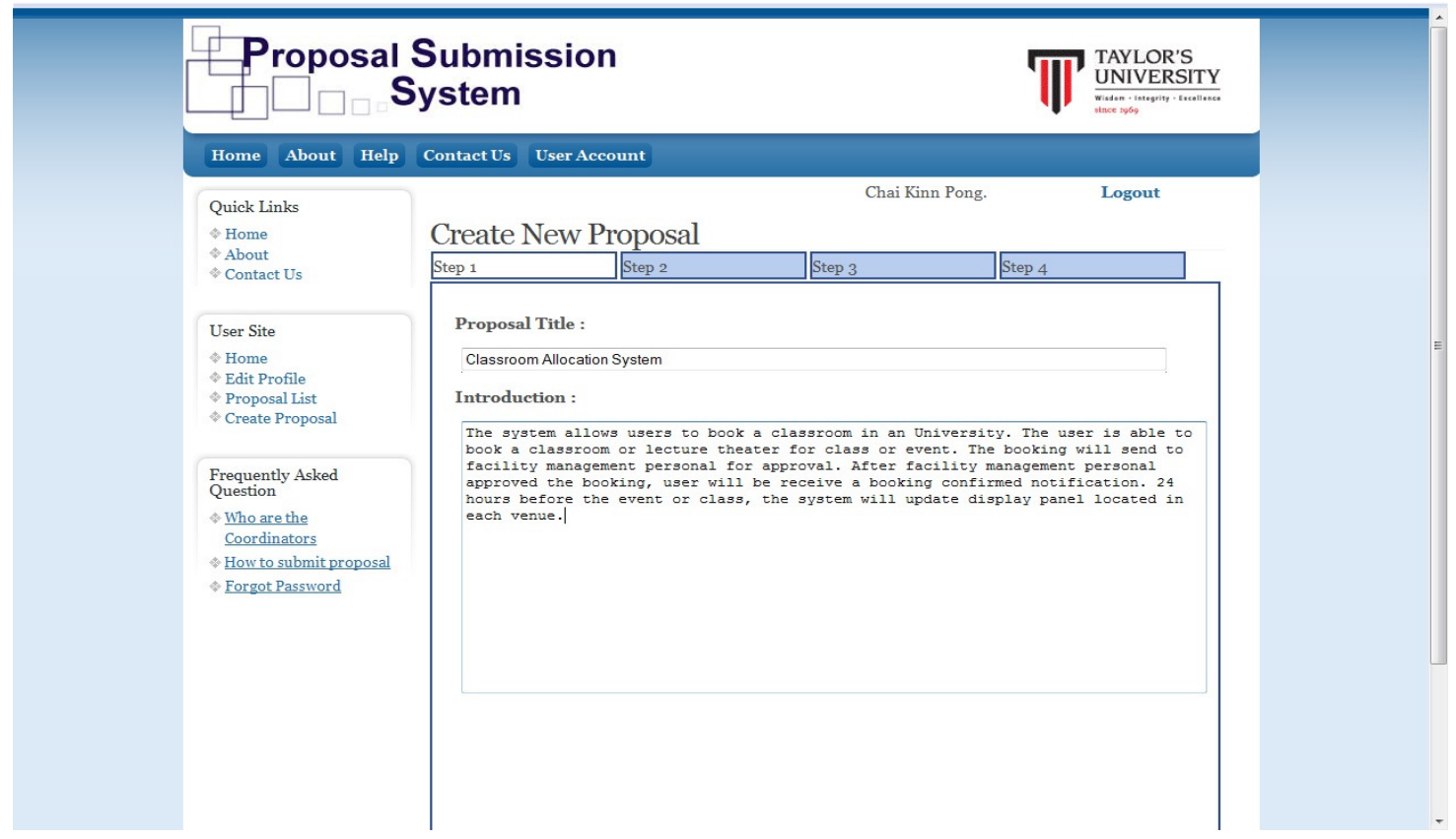

Figure 6 (Create new proposal) 
International Journal of Web \& Semantic Technology (IJWesT) Vol.2, No.2, April 2011

\section{$\underline{\text { Coordinator }}$}

Coordinators are academic staff which will receive, view, evaluate and reply to participant's proposal submitted. Coordinators will be able to do followings:

- a user home page which consist of coordinator details, proposal statistics, and a number of proposal received;

- user profile page, allow coordinator to change and update their information, include changing email and password;

- proposal list, a list of proposal received by coordinator, sorted with creation date of receival and with status of proposal shown;

- notification upon proposal received;

- view a proposal received by coordinator;

- approve or reject a proposal with date of response, if reject with the reason of rejecting the proposal;

- comment and reply on a proposal received;

- report submission or participant.

Coordinator involves in response to proposal submissions, either approve, reject or comment on proposal received. Other than user profile management, report submission or participant is an extra feature for academic staffs to tackle spams and irresponsible users.

\section{System Administrator}

Admin will be personal which in charge of managing the Proposal Submission System. Administrative rights and functionality provided to administrator includes:

- view statistics on usage of the system;

- approve coordinator's registration;

- remove user from the system;

- Modify system settings of the system.

System admin are not directly involved in the system as a user, they are responsible to manage the system. Future iteration of the system might include reporting function to provide statistics based on administrator selected criteria. 
International Journal of Web \& Semantic Technology (IJWesT) Vol.2, No.2, April 2011

\section{Related Proposal Submission System}

There are different exiting Proposal Submission System on Internet. As stated in some of the major Proposal Submission Systems are as follows:

EPSS: The EPSS (Electronic Proposal Submission Service) allows Proposers to create their proposal completely on-line without downloading or saving anything on their local personal computer. [10] The EPSS service is available via the Internet as a Web based application

CRMA: The CARMA Proposal System. This system is used to propose for time on the CARMA array during TAC-approved proposal calls. [11] User can work on proposals and save partial and draft results, and come back later to edit and finish your proposals.

\section{ADVANCED \& UNIQUE FEATURES}

Proposal Submission System had put in place several features other than the general system requirements. These features are to further improve the process of academic proposal submission and approval.

Proposal template provides functionality to coordinators to create format or templates for proposal submission, specify requirements for proposal with a short description. By default, the system uses a summarized system proposal, which consist of 5 sections. With the feature of proposal template, coordinators will be able to specify requirements needed for proposals of different purposes. Coordinators and participants will be able to view all templates created in the system. Creation of proposal will include a step where participants will choose the specific template to be use for his/her proposal.

Another functionality is the implementation of proposal forwarding and submission evaluation of multiple coordinator. This function allows coordinators to forward a proposal to another coordinator, sharing the proposal evaluation right with another coordinator. Proposal forwarding require original coordinator to specify different option: forward and share evaluation rights of the proposal with partner coordinator; send the proposal to another coordinator, allowing read only access to proposal; forward the proposal to second coordinator and give up all evaluation rights to second coordinator.

The Proposal Submission System is implemented in education institution for academic purposes. An example database help to further guide the participants on writing proposal. This feature with the implementation of Proposal Template, coordinator will be able to pick an existing received proposal to be an example for the particular template, or a coordinator will be able to create the example for the proposal template. The example database aim to work as a resource to locate references when participants are writing new proposal for submissions.

Not only proposal forwarding between coordinators, multiple participants may create a proposal as a group. A proposal sharing module is in place to ease group works, in a situation where several were grouped into team to produce a proposal for possible system development or research paper. The group option allows updates on the proposal to notify all member of the 
International Journal of Web \& Semantic Technology (IJWesT) Vol.2, No.2, April 2011

group, including coordinator comments on proposal and approval of proposal. Proposal belong to a group is editable for all group members.

\section{CONCLUSION}

The Proposal Submission System has opened up vast amount of possibilities in providing better and more flexible solution with regards to submission of proposals in comparison to traditional approaches. The Proposal Submission System as described in this paper uses a content management system approach. The system is adorned with a centralized and seamless console

with an array and options for users to maneuver within the system. The system which has been rolled out has benefits in store for both academics and students alike. The system has proven to be highly intuitive and seamless among others to withstand the demands of proposals and its related issues. The developers have put together a well-thought out application which not only automates the manual process but is highly scalable to suit future requirements and other challenges which may arise. It is worth mentioning that the system is not merely automation of manual tasks per se but it addressed concerns plaguing most other manual submission mechanism with regards to compliance with process workflow which the authors feel is one of the major hallmarks of the system.

Having said that, there are provisions for further development. The system for instance could be revised to produce a conference management system. It could also be twigged to produce an assignment submission system. More cold be done with regards to the security aspects of the system. For instance, once could introduce a two-pronged authentication mechanism to improve the integrity of submissions. Further work could be done with regards to reporting with more dynamic reporting formats introduced. Another aspect which is worth considering is with regards to project management. For instance, the system could also be fine-tuned to manage projects and its related communications. It could be injected with an SMS facility and the ability to embed reminder and alerts.

\section{REFERENCES:}

[1] Dr. Margaret Procter, (2009). “The Academic Proposal”, University of Toronto

Available: http://www.writing.utoronto.ca/advice/specific-types-of-writing/academic-proposal

[2] David Jones, Sandy Behrens. (2003) “Online Assignment Submission: An Evolutionary Tale”, in 36th Annual Hawaii International Conference on System Sciences vol. 5, no. 5, pp. 156c

Available: http://davidtjones.wordpress.com/publications/online-assignment-submission-an-evolutionary-tale/

[3] Doug L. Simpson.(2005) “Content for One: Developing a Personal Content Management System”, in SIGUCCS November, pp 338-342

[4] Charles Kiran.(2010) "The Web Development Industry Is Expected To Grow Over 20 By 2010. Internet: http://www.articler.com/23205/The-Web-Development-Industry-Is-Expected-To-Grow-Over-20-By-2010.html,

[5] Nils-Erik Frantzell. .(2004).“Install XAMPP for easy, integrated development. Internet:

http://www.ibm.com/developerworks/linux/library/l-xampp/, Nov. 30, 2004 [Oct 28, 2010]"

[6] W3School "JavaScript. Internet: http://www.w3schools.com/js/js_intro.asp, 
International Journal of Web \& Semantic Technology (IJWesT) Vol.2, No.2, April 2011

[7] Jevon Wright, Jens Dietrich. (2008) "Survey of Existing Language to Model Interactive Web Applications", fifth Asia-Pacific conference on Conceptual Modeling, pp 113-123

[8] Reuven M. Lerner.(2009) “At the Forge - jQuery”, in Linux Journal,

[9] Web development methodology, (2009) http://hyperlink.com.au/UserFiles/File/web-methodology.pdf

[10] The EPSS (Electronic Proposal Submission Service), (2007), https://www.epss-fp7.org/epss/welcome.jsp

[11] CARMA Proposal System, (2009), http://carma-prop.ncsa.uiuc.edu/proposals/ 